\title{
Heat-Evoked Activation of the Ion Channel, TRPV4
}

\author{
Ali Deniz Güler, ${ }^{1}$ Hyosang Lee, ${ }^{1}$ Tohko lida, ${ }^{2}$ Isao Shimizu,, ${ }^{1,3}$ Makoto Tominaga, ${ }^{2}$ and Michael Caterina \\ ${ }^{1}$ Departments of Biological Chemistry and Neuroscience, Johns Hopkins University School of Medicine, Baltimore, \\ Maryland 21205, 2Department of Physiology, Mie University School of Medicine, Tsu/Mie 514-8507, Japan, and \\ 3Dainippon Pharmaceutical Company, Limited, Suita/Osaka 564-0053, Japan
}

The mammalian nervous system constantly evaluates internal and environmental temperatures to maintain homeostasis and to avoid thermal extremes. Several members of the transient receptor potential (TRP) family of ion channels have been implicated as transducers of thermal stimuli, including TRPV1 and TRPV2, which are activated by heat, and TRPM8, which is activated by cold. Here we demonstrate that another member of the TRP family, TRPV4, previously described as a hypoosmolarity-activated ion channel, also can be activated by heat. In response to warm temperatures, TRPV4 mediates large inward currents in Xenopus oocytes and both inward currents and calcium influx into human embryonic kidney 293 cells. In both cases these responses are observed at temperatures lower than those required to activate TRPV1 and can be inhibited reversibly by ruthenium red. Heat-evoked TRPV4-mediated responses are greater in hypo-osmotic solutions and reduced in hyperosmotic solutions. Consistent with these functional properties, we observed TRPV4 immunoreactivity in anterior hypothalamic structures involved in temperature sensation and the integration of thermal and osmotic information. Together, these data implicate TRPV4 as a possible transducer of warm stimuli within the hypothalamus.

Key words: TRPV4; OTRPC4; VR-OAC; VRL-2; TRP12; heat; ion channel; thermotransduction; osmolarity; hypothalamus
The ability to detect changes in environmental and body temperatures is critical for mammalian survival. In the peripheral nervous system skin heating and cooling are detected by thermosensitive neurons tuned to respond over distinct temperature ranges. For example, warm thermoreceptors detect modest skin temperature increases $\left(34-42^{\circ} \mathrm{C}\right)$, whereas heat nociceptors detect painfully hot temperatures $\left(>42^{\circ} \mathrm{C}\right.$ ) (Raja et al., 1999). The CNS also contains temperature-sensitive neurons, most notably in the preoptic/anterior hypothalamus, that are activated specifically by local warming or cooling and simultaneously receive thermally related input from the periphery (Boulant, 2000). Although the molecular basis of temperature sensation remains poorly understood, several ion channels have been identified that are expressed in distinct subsets of peripheral sensory neurons and that can be activated by changes in ambient temperature. Among these are members of the transient receptor potential (TRP) family, including TRPV1 (VR1) (Caterina et al., 1997) and TRPV2 (VRL-1) (Caterina et al., 1999), which are activated by temperatures $>40$ and $>50^{\circ} \mathrm{C}$, respectively, and the distantly related protein TRPM8 (CMR1) (McKemy et al., 2002; Peier et al., 2002), which is activated by cool temperatures $\left(<25^{\circ} \mathrm{C}\right)$. These findings raise the possibility that the transduction of thermal stimuli might be observed among other TRP family members.

TRPV4 (OTRPC4/VR-OAC/VRL-2/TRP 12) is a nonselective cation channel that shares $\sim 40 \%$ amino acid identity with

\footnotetext{
Received Feb. 15, 2002; revised May 20, 2002; accepted May 20, 2002.

This work was supported by American Cancer Society Grant RGS-01-063-01CSM; by awards from the W. M. Keck Foundation, Searle Scholars Program, and Arnold and Mabel Beckman Foundation and a gift from Dainippon Pharmaceuticals to M.J.C.; and by grants from the Ministry of Education, Culture, Sports and Technology, Japan to M.T. We thank J. Wang and R. Evans for technical assistance and P. Coulombe, D. Johns, M. Nealen, and C. Montell for valuable suggestions.

Correspondence should be addressed to M. J. Caterina, Departments of Biological Chemistry and Neuroscience, 725 North Wolfe Street, Johns Hopkins University School of Medicine, Baltimore, MD 21205. E-mail: caterina@jhmi.edu.

Copyright (C) 2002 Society for Neuroscience $0270-6474 / 02 / 226408-07 \$ 15.00 / 0$
}

TRPV1 (Liedtke et al., 2000; Strotmann et al., 2000; Wissenbach et al., 2000; Delany et al., 2001). Expression of TRPV4 protein has been demonstrated in epithelial cells of the renal distal convoluted tubule, trachea, and submucosal glands, in neutrophils, and in autonomic nerve fibers (Delany et al., 2001). In situ hybridization studies also have revealed TRPV4 mRNA expression in hair cells of the inner ear, peripheral sensory ganglia, and osmoregulation-related brain structures, including the vascular organ of the lamina terminalis and the hypothalamic median preoptic region (MnPO) (Liedtke et al., 2000; Schumacher et al., 2000). Consistent with this localization pattern, heterologously expressed TRPV4 can be activated by hypotonic solutions, suggesting that it serves as a sensor for osmolarity and/or mechanical stretch (Liedtke et al., 2000; Strotmann et al., 2000; Wissenbach et al., 2000; Delany et al., 2001). Recently, it was demonstrated that TRPV4 also can be activated by certain phorbol derivatives (Watanabe et al., 2002). Although one previous study has shown that ambient temperature can influence the magnitude of the TRPV4 response to hypo-osmolarity (Liedtke et al., 2000), several studies have failed to detect any activation of TRPV4 by acute changes in ambient temperature (Liedtke et al., 2000; Strotmann et al., 2000; Delany et al., 2001). Here we demonstrate by using two different expression systems that TRPV4 is activated by warm temperatures and that this response is influenced by osmolarity. Moreover, we demonstrate by using immunohistochemistry that TRPV4 protein is expressed in the preoptic/ anterior hypothalamus. Together, these data suggest a possible role for TRPV4 in thermosensation and/or thermoregulation.

\section{MATERIALS AND METHODS}

Molecular biology. An oligonucleotide hybridization probe [5'-taagtaccccgtggtcttc-3', nucleotides (nt) $2160-2178$ of the rat vanilloid receptorrelated osmotically activated channel (VR-OAC) coding region] (Liedtke et al., 2000) was used to isolate a partial TRPV4 cDNA from a rat dorsal root ganglion cDNA library in pCDNA3 (Invitrogen, Carlsbad, CA). Ligation of a 283 bp 5'-RACE fragment obtained from rat kidney to this 
partial clone resulted in a full-length cDNA containing an open reading frame of $2612 \mathrm{bp}$ identical to that of rat VR-OAC. TRPV1 cDNA in pCDNA3 has been described previously (Caterina et al., 1999). Drosophila TRPL (Xu et al., 1997) and human TRPC3 (Wes et al., 1995) cDNAs in pCDNA3 were gifts of C. Montell (Johns Hopkins University, Baltimore, MD). TRPV1 and TRPV4 cDNAs also were subcloned between the 5'and $3^{\prime}$-untranslated regions of Xenopus $\beta$-globin in $\mathrm{pX} \beta \mathrm{G}$ (gift of P. Agre, Johns Hopkins University). Unless otherwise noted, molecular biology and cell culture reagents were obtained from Invitrogen and chemicals from Sigma (St. Louis, MO) or Fisher (Pittsburgh, PA).

Oocyte expression system and electrophysiology. TRPV1 and TRPV4 cDNAs in pCDNA3 or pX $\beta \mathrm{G}$ were transcribed in vitro with $\mathrm{T} 7$ or T3 RNA polymerase (Epicentre Technologies, Madison, WI) after linearization with XbaI. Stage V Xenopus laevis (Nasco, Modesto, CA) oocytes were defolliculated with collagenase (Worthington, Lakewood, NJ) and injected the next day with 1-50 ng of TRPV1 or TRPV4 cRNA in $50 \mathrm{nl}$ of water. Oocytes were subjected to two-electrode voltage clamp $\left(E_{\mathrm{h}}=\right.$ $-40 \mathrm{mV}$ ) on days 2-7 after injection via a TEV-200A amplifier (Dagan, Minneapolis, MN), PowerLab A/D converter (AD Instruments, Mountain View, CA), and $3 \mathrm{M} \mathrm{KCl-filled} \mathrm{electrodes} \mathrm{with} \mathrm{a} \mathrm{resistance} \mathrm{of} \mathrm{0.4-2}$ $\mathrm{M} \Omega$. Normal (210 mOsm) bath solution composition (in $\mathrm{mM}$ ) was 96 $\mathrm{NaCl}, 2 \mathrm{KCl}, 1 \mathrm{MgCl}_{2}, 0.1 \mathrm{CaCl}_{2}$, and 5 HEPES, adjusted to $\mathrm{pH} 7.4$ with $\mathrm{NaOH}$. Hyperosmotic (410 mOsm) bath solution was supplemented with $200 \mathrm{~mm}$ mannitol. Perfusion rate was $1 \mathrm{ml} / \mathrm{min}$. Heat ramps from 27 to $45^{\circ} \mathrm{C}$ in $15 \mathrm{sec}$ were applied by preheating the perfusate with a Peltier controller (Dagan) and were monitored with a thermistor (Physitemp, Clifton, NJ) within $2 \mathrm{~mm}$ of the oocyte. All procedures involving the care and use of animals were performed in accordance with institutional guidelines.

Mammalian cell culture and calcium imaging. Human embryonic kidney (HEK) 293/T-antigen cells maintained in DMEM/10\% fetal bovine serum/penicillin/streptomycin/L-glutamine were transiently transfected with $2 \mu \mathrm{g}$ of plasmid DNA per $35 \mathrm{~mm}$ dish (including $125 \mathrm{ng} /$ well green fluorescent protein (GFP) cDNA in pCDNA3) by using Lipofectamine2000. Cells were replated onto polyornithine-coated glass coverslips after $24 \mathrm{hr}$ and subjected to calcium imaging $24 \mathrm{hr}$ later. Stable transformant TRPV1, TRPV4, and pcDNA3 cell lines were generated by selection of transfected HEK 293 cells lacking T-antigen (gift of J. Nathans, Johns Hopkins University) with $500 \mu \mathrm{g} / \mathrm{ml} \mathrm{G418.} \mathrm{Resistant}$ clones were screened for TRPV1 or TRPV4 surface expression by immunofluorescence. The normal $(290 \mathrm{mOsm})$ bath solution contained (in $\mathrm{mM}$ ) $130 \mathrm{NaCl}, 3 \mathrm{KCl}, 2.5 \mathrm{CaCl}_{2}, 0.6 \mathrm{MgCl}_{2}, 10 \mathrm{HEPES}, 1.2$ $\mathrm{NaHCO}_{3}$, and 10 glucose, adjusted to $\mathrm{pH} 7.45$ with $\mathrm{NaOH}$. For the experiment shown in Figure 5, this solution was adjusted to $300 \mathrm{mOsm}$ with mannitol. The $400 \mathrm{mOsm}$ solution was prepared by adding an additional $100 \mathrm{~mm}$ mannitol. For the $250 \mathrm{mOsm}$ solution $\mathrm{NaCl}$ was reduced to $105 \mathrm{~mm}$. The $300 \mathrm{mOsm}$ low-sodium solution $(300 / \mathrm{M})$ was prepared by the addition of mannitol to the $250 \mathrm{mOsm}$ solution. Osmolarities were measured by a vapor pressure osmometer (Wescor, Logan, UT). For calcium-free solution the $\mathrm{CaCl}_{2}$ was replaced with $10 \mathrm{~mm}$ EGTA. Cells were loaded with fura- $2 \mathrm{AM}\left(10 \mu \mathrm{M} ; 37^{\circ} \mathrm{C}\right.$ for $\left.40 \mathrm{~min}\right)$ in normal bath solution containing $0.02 \%$ pleuronic acid (Molecular Probes, Eugene, OR). Ratiometric calcium imaging was performed with an inverted fluorescence microscope (Nikon, Melville, NY), excitation filter changer (Sutter, Novato, CA), and cooled CCD camera (Roper, Tucson, AZ). Paired images (340 and $380 \mathrm{~nm}$ excitation, $510 \mathrm{~nm}$ emission) were collected every $2 \mathrm{sec}$ with RatioTool software (ISee Imaging, Raleigh, NC). Transiently transfected cells were identified on the basis of green fluorescent protein expression. Heating was achieved as for oocyte recording and monitored with a thermistor placed within $2 \mathrm{~mm}$ of the microscopic field. Target temperatures were reached within 25-45 sec. Fura ratios were calculated from 30 to 50 cells per coverslip, and average ratios from multiple independent coverslips were used to calculate the sample mean \pm SEM. Unless otherwise indicated, statistical comparisons were made with paired or unpaired Student's $t$ tests.

Patch-clamp electrophysiology. Whole-cell patch-clamp recordings were performed on stable transformant TRPV1, TRPV4, and pCDNA3 cell lines as described previously (Caterina et al., 1997). The standard bath solution contained (in mM) $140 \mathrm{NaCl}, 5 \mathrm{KCl}, 2 \mathrm{MgCl}_{2}, 2 \mathrm{CaCl}_{2}, 10$ HEPES, and 10 glucose, pH 7.4 (adjusted with $\mathrm{NaOH}$ ). The pipette solution contained (in mM) $140 \mathrm{KCl}, 5$ EGTA, and 10 HEPES, pH 7.4 (adjusted with $\mathrm{KOH}$ ). When examining the heat-evoked current responses, we increased bath temperature with a preheated solution at a rate of $\sim 0.2^{\circ} \mathrm{C} / \mathrm{sec}$. When heat-activated currents began to inactivate, the perfusate was changed to a $22^{\circ} \mathrm{C}$ solution. Chamber temperature was monitored within $\pm 0.1^{\circ} \mathrm{C}$ with a thermocouple placed within $4 \mathrm{~mm}$ of the patch-clamped cell. Holding potential was $-60 \mathrm{mV}$. For currentvoltage analysis, voltage-ramp pulses $(-100$ to $+100 \mathrm{mV})$ were applied over $700 \mathrm{msec}$. Current responses obtained before heat application were subtracted from current responses at $40^{\circ} \mathrm{C}$ (TRPV4, pCDNA3) or $45^{\circ} \mathrm{C}$ (TRPV1).

Antibody generation and immunohistochemistry. Rabbits were immunized with a peptide corresponding to the TRPV4 C terminus (CDGHQQGYAPKWRAEDAPL) coupled to hemocyanin (Strategic Biosolutions, Newark, DE). TRPV4 antibodies were purified from serum by peptide chromatography (Ultralink, Pierce, Rockford, IL). Antibody reactivity and specificity were confirmed by the detection of discrete protein bands in immunoblots of TPV4-transfected HEK 293 cell extracts, but not extracts from cells transfected with pCDNA3, TRPV1, or TRPV2. Adult male Sprague Dawley rats anesthetized with ketamine $(100 \mathrm{mg} / \mathrm{kg}$, i.p.) and xylazine $(10 \mathrm{mg} / \mathrm{kg}$, i.p.) were perfused with PBS and then with ice-cold $4 \%$ formaldehyde/PBS. Tissues were fixed overnight, cryoprotected (for $48 \mathrm{hr}$ in $30 \%$ sucrose/PBS), embedded in OCT (Ted Pella, Redding, CA), and cryosectioned at 16-30 $\mu \mathrm{m}$. Immunostaining was performed as described previously (Caterina et al., 1999), using affinity-purified anti-TRPV4 or anti-TRPV1 $(1 \mu \mathrm{g} / \mathrm{ml})$, followed by biotinylated goat anti-rabbit IgG and nickel-enhanced diaminobenzidine detection (Vector, Burlingame, CA) or Cy3-conjugated goat anti-rabbit IgG (Jackson ImmunoResearch, West Grove, PA). Staining specificity was confirmed by ablation of the signal after the incubation of antibodies with TRPV4 peptide-conjugated resin, but not with VR1 peptideconjugated resin.

\section{RESULTS}

\section{TRPV4 mediates heat-evoked currents in Xenopus oocytes}

To evaluate TRPV4 as a possible heat transducer, we examined its thermal sensitivity in the Xenopus oocyte expression system. Within 2-7 d the oocytes injected with complementary RNA (cRNA) encoding TRPV4 exhibited large inward currents after being heated to $45^{\circ} \mathrm{C}$ within $15 \mathrm{sec}(-1023 \pm 103 \mathrm{nA}$ at $-40 \mathrm{mV}$; $n=48$ ) (Fig. $1 B$ ). Minimal heat-evoked currents were observed in water-injected control oocytes $(-33 \pm 10 \mathrm{nA} ; n=28 ; p<$ 0.0001 ) (Fig. 1A). With optimization of TRPV4 expression conditions the heat-evoked responses were observed in 87 of 90 TRPV4-injected oocytes. These currents could be produced repeatedly in the same oocyte, with an amplitude decrement of only $17 \pm 10 \%$ over six consecutive challenges (Fig. $1 C$, inset). As with TRPV4 responses evoked by hypotonic stimuli (Strotmann et al., $2000)$, the inclusion of ruthenium red $(100 \mathrm{nM})$ in the perfusate during the third and fourth heat applications significantly inhibited heat-evoked currents ( $87 \pm 2 \%$ reduction; $n=4 ; p<0.001)$ (Fig. $1 C$, inset), and these responses recovered only partially after a $10 \mathrm{~min}$ washout. Neither the removal of extracellular calcium ions nor the addition of 4,4'-diisothiocyanostilbene2,2'-disulfonic acid (DIDS; $500 \mu \mathrm{M}$ ) reduced the amplitude of heat-evoked TRPV4 responses (data not shown), indicating that current contamination by calcium-activated chloride flux was minimal. As described previously (Caterina et al., 1999), oocytes microinjected with cRNA encoding TRPV1 also exhibited robust inward currents at elevated temperatures, with the initial heatevoked response appearing at slightly higher temperatures (40$42^{\circ} \mathrm{C}$ ) than subsequent responses $\left(36-38^{\circ} \mathrm{C}\right)$ (Fig. $\left.1 D\right)$. By comparison, typical heat-evoked currents in oocytes expressing TRPV4 often were detectable with temperature elevation above $\sim 27^{\circ} \mathrm{C}$ and always commenced at temperatures lower than those required to activate TRPV1. TRPV4-expressing oocytes also exhibited a shallower temperature-response profile than cells expressing TRPV1. Sustained heating beyond $42^{\circ} \mathrm{C}$ typically resulted in a decline in the amplitude of the TRPV4 heat-evoked current response even as the temperature continued to rise (Fig. $1 B-D)$. Despite this desensitization phenomenon, however, fluc- 


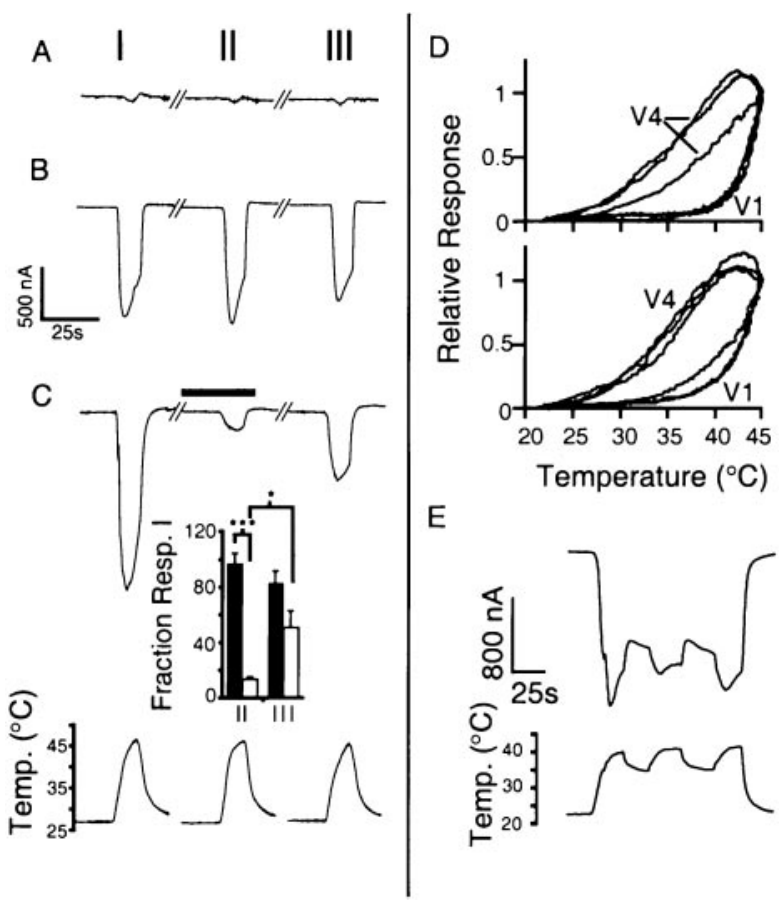

Figure 1. TRPV4 mediates heat-evoked currents in Xenopus oocytes. $A-C$, Two-electrode voltage-clamp recordings. Shown are representative second $(I)$, fourth $(I I)$, and sixth (III) heat-evoked current responses to consecutive heat ramps (from 27 to $45^{\circ} \mathrm{C}$ in $15 \mathrm{sec}$, indicated on the scale at the bottom) for water-injected control oocytes $(A)$ and oocytes injected with TRPV4 cRNA $(B, C)$. In $C$, ruthenium red (horizontal bar, $100 \mathrm{~nm}$ ) was added $30 \mathrm{sec}$ before the third heat application and washed away for $10 \mathrm{~min}$ before the fifth heat application. Inset, Amplitudes of responses II and III relative to response I with (open columns) or without (filled columns) ruthenium red treatment. Data represent the mean $\pm \mathrm{SEM} ; n=4\left({ }^{*} p<\right.$ 0.01 ; ${ }^{* *} p<0.001$; unpaired $t$ test). $D$, Representative temperature response profiles evoked by an initial heat stimulus (top) and a second heat stimulus (bottom) in oocytes expressing TRPV4 (V4) or TRPV1 (V1). Heat stimulus ramps went from 22 to $45^{\circ} \mathrm{C}$ in $15 \mathrm{sec}$. Currents were normalized to the amplitude at $45^{\circ} \mathrm{C}$. $E$, Effect of suprathreshold temperature fluctuations (bottom) on representative TRPV4-mediated current response (top).

tuations in temperature between 36 and $42^{\circ} \mathrm{C}$ evoked correspondingly fluctuating current responses (Fig. $1 E$ ), as described previously for TRPV1 (Tominaga et al., 1998). These results suggest that TRPV4 responds dynamically to temperature changes within the physiological range.

\section{TRPV4 mediates heat-evoked calcium influx in HEK 293 cells}

We next examined heat-evoked responses in HEK 293 cells transiently transfected with TRPV4 cDNA under the control of a cytomegalovirus promotor. TRPV4 expression in these mammalian cells was evident from their elevated basal $\left[\mathrm{Ca}^{2+}\right]_{i}$ (Fig. $2 A, B)$ and was confirmed by immunofluorescence microscopy (data not shown). As reported previously (Liedtke et al., 2000; Strotmann et al., 2000), TRPV4-expressing cells exhibited a reversible increase in $\left[\mathrm{Ca}^{2+}\right]_{\mathrm{i}}$ after exposure to a hypo-osmotic medium (250 mOsm), whereas no such response was observed in cells expressing the control vector, pCDNA3 (Fig. $2 A$ ). In response to a heat stimulus $\left(25-40^{\circ} \mathrm{C}\right.$ in $\left.\sim 40 \mathrm{sec}\right)$ control cells exhibited only a modest rise in $\left[\mathrm{Ca}^{2+}\right]_{\mathrm{i}}(0.079 \pm 0.018$ fura ratio units, RU; $n=5$ ) (Fig. $2 B, C$ ). In contrast, cells transfected with TRPV4 exhibited a significantly larger rise $(0.251 \pm 0.033 \mathrm{RU}$; $n=12 ; p<0.001)$ once the temperature exceeded $\sim 34^{\circ} \mathrm{C}$. A
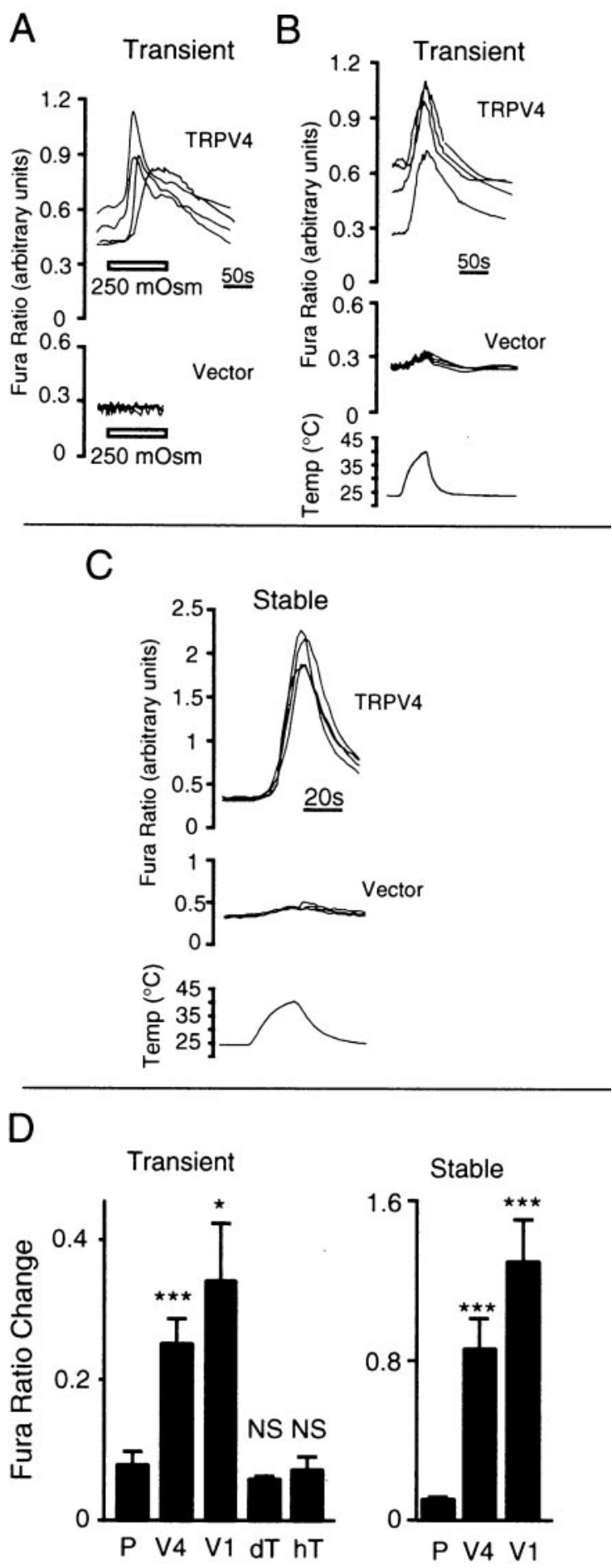

Figure 2. TRPV4 mediates heat-evoked calcium influx in HEK 293 cells. $A$, Relative $\left[\mathrm{Ca}^{2+}\right]_{\mathrm{i}}$ (indicated by ratio of fura- 2 emission at $340 / 380 \mathrm{~nm}$ excitation) in HEK 293 cells transiently transfected with TRPV4 or control vector (four each) after a reduction in osmolarity from 290 to 250 mOsm (horizontal bars) at $24^{\circ} \mathrm{C} . B, C$, Relative $\left[\mathrm{Ca}^{2+}\right]_{\mathrm{i}}$ in HEK 293 cells transiently $(B)$ or stably $(C)$ transfected with TRPV4 or control vector (four each) during a heat stimulus from 24 to $40^{\circ} \mathrm{C}$ in $\sim 40 \mathrm{sec} . D$, Comparison of heat-evoked $\left(40^{\circ} \mathrm{C}\right)$ increases in fura ratio among cells transiently transfected with control vector $(P ; n=5)$, TRPV4 (V4;n= 12), TRPV1 $(V 1 ; n=3)$, Drosophila TRPL $(d T ; n=3)$, or human TRPC3 $(h T ; n=4)$ or among stable transformants generated with control vector $(P ; n=8)$, TRPV4 $(V 4 ; n=15)$, or TRPV1 $(V 1 ; n=13)$. Data represent the mean \pm SEM of the indicated number of coverslips. Comparisons are with controls. ${ }^{*} p<0.05 ;{ }^{* * *} p<0.001 ; N S$, not significant; unpaired $t$ test. 
heat-evoked response of similar amplitude was observed in cells transfected with TRPV1 $(0.341 \pm 0.08 \mathrm{RU} ; n=3)$, but not in cells transfected with either of two distantly related channel proteins, Drosophila TRPL (0.059 $\pm 0.004 \mathrm{RU} ; n=3)$ or human TRPC3 $(0.07 \pm 0.02 \mathrm{RU} ; n=4)$ (Fig. $2 D)$. This is despite the fact that both channels have been shown to exhibit constitutive activity in mammalian cell lines (Xu et al., 1997; Zitt et al., 1997) and that, like TRPV4-transfected cells, TRPL-transfected cells exhibited baseline elevations in $\left[\mathrm{Ca}^{2+}\right]_{i}$ (data not shown).

To rule out the possibility that the heat-evoked responses observed in TRPV4-expressing cells were an artifact of the transient transfection method, we generated a stable HEK 293 cell line expressing this protein. Stable TRPV4 transformants had a baseline $\left[\mathrm{Ca}^{2+}\right]_{\mathrm{i}}$ similar to that of control cells but exhibited an average heat-evoked $\left[\mathrm{Ca}^{2+}\right]_{\mathrm{i}}$ increase that was 3.4-fold as large as that observed in cells transiently transfected with TRPV4 (Fig. 2C) and comparable with that exhibited by stable TRPV1 transformants. Like responses to other stimuli (Liedtke et al., 2000; Strotmann et al., 2000; Watanabe et al., 2002), heat-evoked $\left[\mathrm{Ca}^{2+}\right]_{\mathrm{i}}$ increases mediated by TRPV4 could be inhibited reversibly by ruthenium red (200 nм; $75 \pm 5 \%$ reduction; $n=3 ; p<0.05$ vs control) or by the removal of calcium from the extracellular medium $(90 \pm 3 \%$ reduction; $n=3 ; p<0.01$ vs control) (Fig. $3 A$ ). In contrast, pretreatment of TRPV4-expressing HEK 293 cells with thapsigargin $(10 \mu \mathrm{M})$ and 1-[ $\beta$-(3-[4-methoxyphenyl]propoxy)-4-methoxyphenethyl]-1H-imidazole hydrochloride $(20 \mu \mathrm{M})$ failed to reduce the amplitude of the heat-evoked calcium response (data not shown). Together, these findings suggest that the observed rise in $\left[\mathrm{Ca}^{2+}\right]_{\mathrm{i}}$ results predominantly from the heat-evoked activation of calcium influx through TRPV4 and that the release of calcium from intracellular stores does not appear to contribute significantly to this response.

Consistent with the oocyte findings, TRPV4 expressed in HEK 293 cells was more sensitive to heat than TRPV1 (Fig. 3B). Although TRPV4-mediated calcium responses could be observed at temperatures as low as $34^{\circ} \mathrm{C}$, TRPV1-mediated responses first became apparent only at temperatures two to four degrees higher, with relatively modest responses below $40^{\circ} \mathrm{C}$. Two additional features of TRPV4 heat responsiveness mirrored those observed in the oocyte system. First, the temperature-response profile exhibited by TRPV4 was shallower than that exhibited by TRPV1. Second, when the ambient temperature was raised from 25 to $37^{\circ} \mathrm{C}$ and maintained for $15 \mathrm{~min}$, the resulting TRPV4mediated calcium response slowly declined to baseline (data not shown). With a subsequent increase to $42^{\circ} \mathrm{C}$, however, a second calcium response was observed that again was significantly larger than that observed in pCDNA3-transformed control cells (Fig. 3C).

\section{TRPV4 mediates heat-evoked currents in HEK 293 cells}

Further evidence for heat-evoked activation of TRPV4 was provided by whole-cell voltage-clamp studies of HEK 293 cells stably expressing this protein. When cells were held at $-60 \mathrm{mV}$, elevations in bath temperature from 25 to $45^{\circ} \mathrm{C}$ over $80 \mathrm{sec}$ resulted in inward currents $(-238 \pm 80 \mathrm{pA} ; n=5)$ significantly larger than any observed in pCDNA3 transformants $(17.8 \pm 26 \mathrm{pA} ; n=5$; $p<0.05$ ) (Fig. 4A). Temperature response profiles revealed a threshold temperature for TRPV4 activation $\left(33.6 \pm 1.8^{\circ} \mathrm{C} ; n=\right.$ 5) similar to that obtained in the calcium-imaging experiments (Fig. 4B). Although the initial portion of heat-evoked TRPV4 responses exhibited steep temperature dependence $\left(Q_{10}=9.9 \pm\right.$ $3.8 ; n=5)$, the responses desensitized at temperatures several degrees above threshold. Current-voltage analyses performed at suprathreshold temperatures revealed a reversal potential for TRPV4-mediated currents near $0 \mathrm{mV}$ (Fig. 4C), consistent with a nonselective cationic current. Although the resulting profiles were outwardly rectifying, as reported for hypo-osmolarity-evoked TRPV4 responses (Liedtke et al., 2000; Strotmann et al., 2000), the degree of rectification was substantially less than that exhibited by heat-evoked currents mediated by TRPV1 (Rectification ratio at \pm $100 \mathrm{mV}$ : TRPV4, $2.08 \pm 0.44$; TRPV1, $18.00 \pm 6.31 ; n=4)$.

\section{Osmolarity modulates the amplitude of heat-evoked TRPV4 responses}

Given that TRPV4 exhibits responsiveness to both heat and hypo-osmolarity and that temperature reportedly influences hypo-osmolarity-evoked responses in TRPV4-expressing cells (Liedtke et al., 2000), we sought to determine whether osmolarity would impact TRPV4 heat responsiveness. We activated TRPV4expressing Xenopus oocytes with two consecutive heat applications in standard isotonic bath solution, followed by a pair of stimuli in hypertonic bath solution (410 mOsm) (Fig. 5A). The increased osmolarity substantially reduced the amplitude of TRPV4-mediated heat-evoked responses $(72 \pm 6 \%$ reduction; $n=4 ; p<0.001)$. This effect was reversible, because the response size returned to normal after reversion to an isotonic bath solution. It was also specific to TRPV4, because TRPV1-mediated responses evoked by heat under hypertonic versus isotonic conditions were indistinguishable from one another.

A similar dependence of heat-evoked activation of TRPV4 on osmolarity was observed in the HEK 293 expression system. Under hyperosmotic conditions (400 mOsm) stable TRPV4 transformants exhibited a reduction in their mean calcium influx response to heat $\left(40^{\circ} \mathrm{C}\right)$ compared with control TRPV4 cells that were heat stimulated at $300 \mathrm{mOsm}(0.10 \pm 0.01 \mathrm{RU}, n=6$ vs $0.47 \pm 0.03 \mathrm{RU}, n=5 ; p<0.0001$ ) (Fig. 5B,C). After restimulation with heat under isotonic conditions, the cells initially heated in the hypertonic solution exhibited larger heat-evoked responses resembling those of isotonic controls (data not shown). A decrease in osmolarity to $250 \mathrm{mOsm}$ (at a constant extracellular sodium concentration) produced a hypo-osmolarity-evoked response that reached a peak within $\sim 2 \mathrm{~min}$. A heat stimulus applied at this point evoked a superimposed calcium response that was significantly larger than that observed at 300 mOsm $(1.54 \pm 0.33 \mathrm{RU}, n=6$ vs $0.59 \pm 0.07 \mathrm{RU}, n=6 ; p<0.05)$. Indeed, even if the hypo-osmolarity-evoked response was allowed to desensitize to baseline before heat challenge (after 5-15 $\mathrm{min}$ ), a large heat-evoked response was still observed (1.23 $\pm 0.25 \mathrm{RU}$; $n=9 ; p<0.05$ vs 300 mOsm response). Together, these data argue strongly that heat and osmolarity can act in concert on TRPV4 to regulate its activity, but that even after the hypoosmolarity-evoked response of TRPV4 has undergone desensitization, substantial responsiveness to heat persists.

\section{TRPV4 is expressed in thermosensory regions of the hypothalamus}

In an attempt to identify anatomical structures in which the coordinate detection of osmolarity and temperature by TRPV4 might be physiologically relevant, we performed TRPV4 immunolocalization in rat tissues. Among a number of brain regions noted to exhibit specific TRPV4 immunoreactivity was the preoptic/anterior hypothalamus, most notably the medial preoptic area (MPA) (Fig. 6A) and MnPO (Fig. 6B). Although we detected TRPV4 mRNA in dorsal root and trigeminal ganglia by 


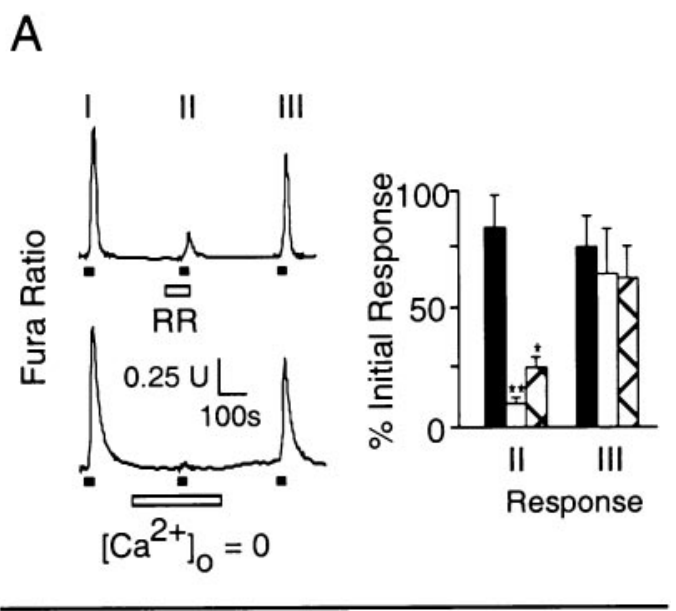

B

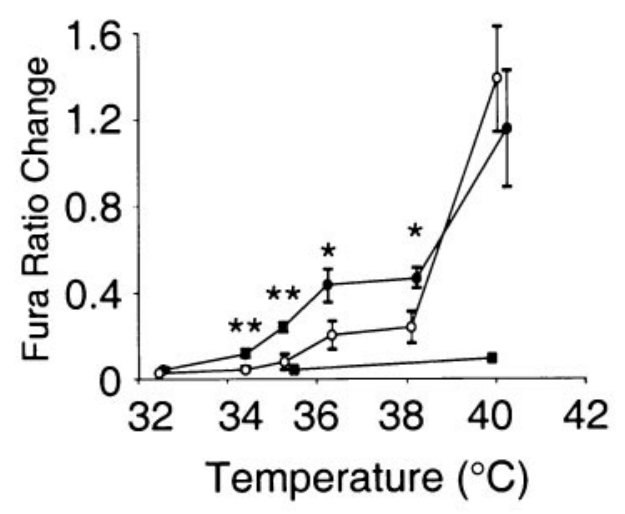

C
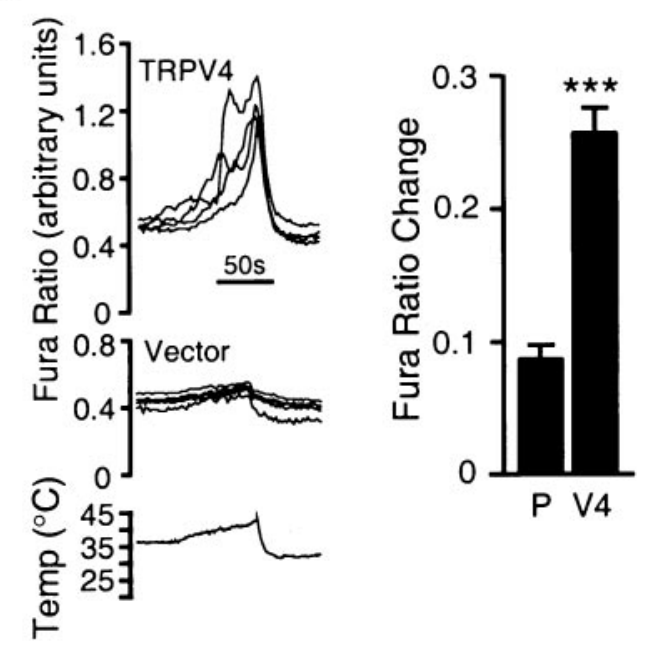

Figure 3. Characterization of heat-evoked calcium responses in TRPV4expressing HEK 293 cells $A$, Reversible inhibition of heat-evoked (ramp to $40^{\circ} \mathrm{C}$; horizontal filled bars) calcium influx in cells stably expressing TRPV4 by ruthenium red $(R R ; 200 \mathrm{nM}$; horizontal open bar, top $)$ or by removal of extracellular calcium (horizontal open bar, bottom). Shown at right are relative amplitudes of heat-evoked responses during ruthenium red (hatched bars; $n=3$ ) or calcium-free (open bars; $n=3$ ) challenge (response II/response I) or after return to normal bath solution (response III/response I). Control ( filled bars; $n=4$ ) represents three consecutive responses in normal bath solution. $B$, Temperature response profiles of heat-evoked calcium responses in cells stably transformed with vector
A
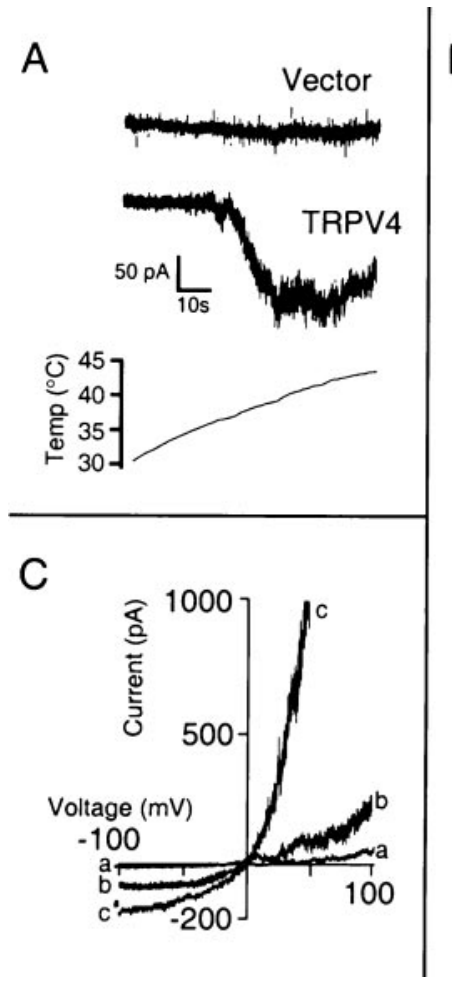

B
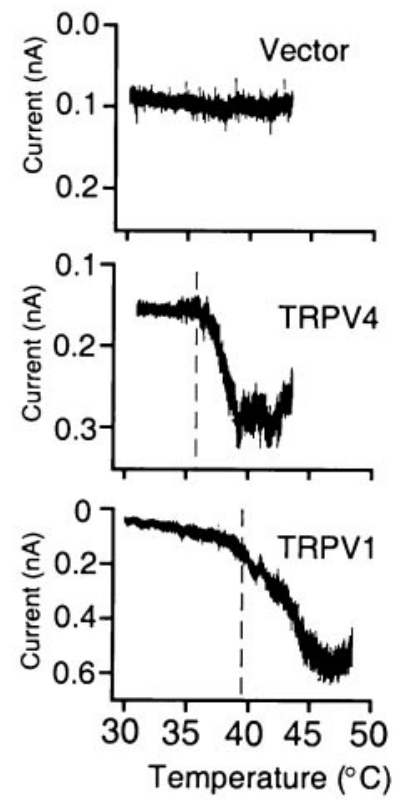

Figure 4. TRPV4 mediates heat-evoked currents in HEK 293 cells. $A$, Representative whole-cell current traces (at $-60 \mathrm{mV}$ ) from HEK 293 cells stably transformed with TRPV4 or control vector during the temperature ramp indicated at the bottom. $B$, Representative temperature-response profiles derived from cells stably transformed with TRPV4, TRPV1, or control vector. $C$, Representative current-voltage relations for heat-evoked responses in cells transformed with TRPV4 $(b)$, TRPV1 $(c)$, or control vector $(a)$. Similar patterns were observed in four cells of each type.

Northern blot and RT-PCR, we observed no specific TRPV4 immunoreactivity in the cell bodies of these ganglia (data not shown). We did, however, observe intense TRPV4 immunoreactivity within suprabasal keratinocytes of plantar skin (Fig. 6C,D).

\section{DISCUSSION}

We have found that heterologously expressed TRPV4 mediates heat-activated current influx in Xenopus oocytes and HEK 293 cells. In oocytes TRPV4 activation becomes apparent at temperatures $>27^{\circ} \mathrm{C}$, whereas in the HEK 293 cells the threshold for activation is $\sim 34^{\circ} \mathrm{C}$. In both systems, therefore, TRPV4 can be activated by modestly elevated (i.e., warm) temperatures lower than those required to activate TRPV1. Prolonged suprathreshold heat stimuli cause the TRPV4-mediated response to desensitize, a phenomenon reported previously for TRPV1 (Caterina et al., 1999). Of potential physiological importance, however, is our observation that, once TRPV4-expressing HEK 293 cells have

( filled squares; $n=3-4$ ), TRPV4 ( filled circles; $n=4-11$ ), or TRPV1 (open circles; $n=4-12$ ). Data represent the mean \pm SEM of the indicated number of independent microscopic fields. $C$, Heat-evoked calcium responses in stable TRPV4 transformants (V4) or vector control cells (vector; $P$ ) after acclimation $(15 \mathrm{~min})$ to $37^{\circ} \mathrm{C}$. Left, Representative traces from four cells of each type. Right, Mean \pm SEM increase in fura ratio $(n=4)$. Data represent the mean \pm SEM of the indicated number of coverslips. Comparisons are with controls $(A, C)$ or between TRPV4 and TRPV1 $(B) .{ }^{*} p<0.05 ;{ }^{* *} p<0.01 ;{ }^{* * *} p<0.001$; unpaired $t$ test. 
A

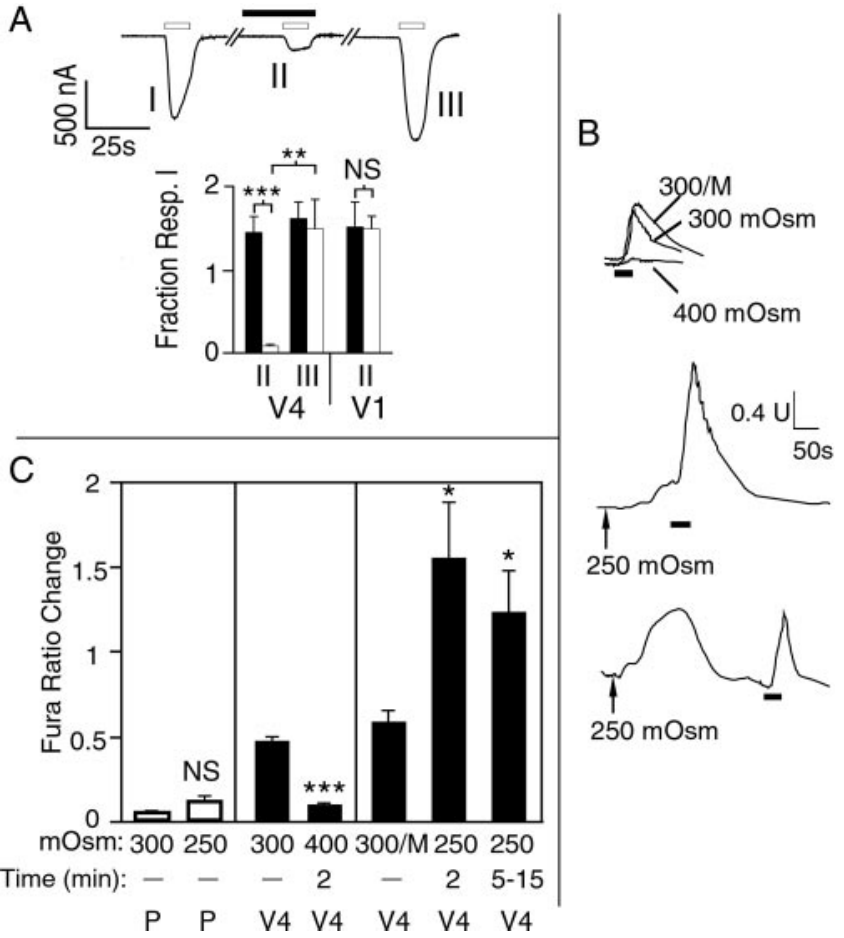

Figure 5. TRPV4-mediated heat-evoked responses are modulated by osmolarity. A, Representative current traces of the second (I), fourth (II), and sixth (III) responses of a TRPV4-expressing oocyte subjected to consecutive heat stimuli $\left(45^{\circ} \mathrm{C}\right.$; horizontal open bars $)$. Hypertonic bath solution (410 mOsm; horizontal filled bar) was applied 1 min before the third heat stimulus, and the oocytes were washed for $2 \mathrm{~min}$ after the fourth heat stimulus. Inset, Amplitudes of heat-evoked responses II and III relative to response I in 410 mOsm-treated (open columns) and untreated ( filled columns) TRPV4- and TRPV1-expressing oocytes. Data represent the mean \pm SEM of four oocytes. $B$, Representative heatevoked $\left(40^{\circ} \mathrm{C}\right.$, horizontal filled bars) calcium influx responses in HEK 293 cells stably expressing TRPV4. Bath solution osmolarity is indicated. The $300 \mathrm{mOsm}$ low-sodium (300/M) and $250 \mathrm{mOsm}$ solutions contained equivalent $\mathrm{NaCl}$ concentrations and differed only by the presence or absence, respectively, of mannitol. In the bottom two traces the experiment was initiated in 300/M solution and was switched to $250 \mathrm{mOsm}$ at the time indicated by the arrows. $C$, Summary of heat-evoked calcium responses at indicated osmolarities for cells stably transformed with control vector $(P$; open columns; $n=3-4$ ) or TRPV4 (V4; filled columns; $n=5-9$ ). Data represent the mean \pm SEM of the indicated number of coverslips. Comparisons in $A$ and $C$ are with the appropriate $300 \mathrm{mOsm}$ or 300/M controls. ${ }^{*} p<0.05 ;{ }^{* *} p<0.01 ;{ }^{* * *} p<0.001$ (NS, not significant; unpaired $t$ test).

been acclimated at $37^{\circ} \mathrm{C}$, TRPV4 remains capable of mediating responses to further increases in ambient temperature.

It is unclear why several other investigators have failed to observe the heat-evoked activation of TRPV4 (Liedtke et al., 2000; Strotmann et al., 2000; Delany et al., 2001). One possible explanation stems from our observation that, in HEK 293 cells transiently expressing TRPV4, a substantial fraction of TRPV4 immunoreactivity resides within an apparently intracellular compartment, whereas in stable TRPV4 transformants nearly all TRPV4 apparently is associated with the plasma membrane (data not shown). The incomplete surface expression of TRPV4 in transiently transfected cells is associated with smaller mean heat-evoked calcium responses than those observed in stable transformants. Moreover, heat stimulation produces a "background" calcium increase in control vector transfected cells that becomes relatively large above $45^{\circ} \mathrm{C}$. Because hypo-osmolarity produces no such background, small heat-evoked responses might have been masked out of pro-

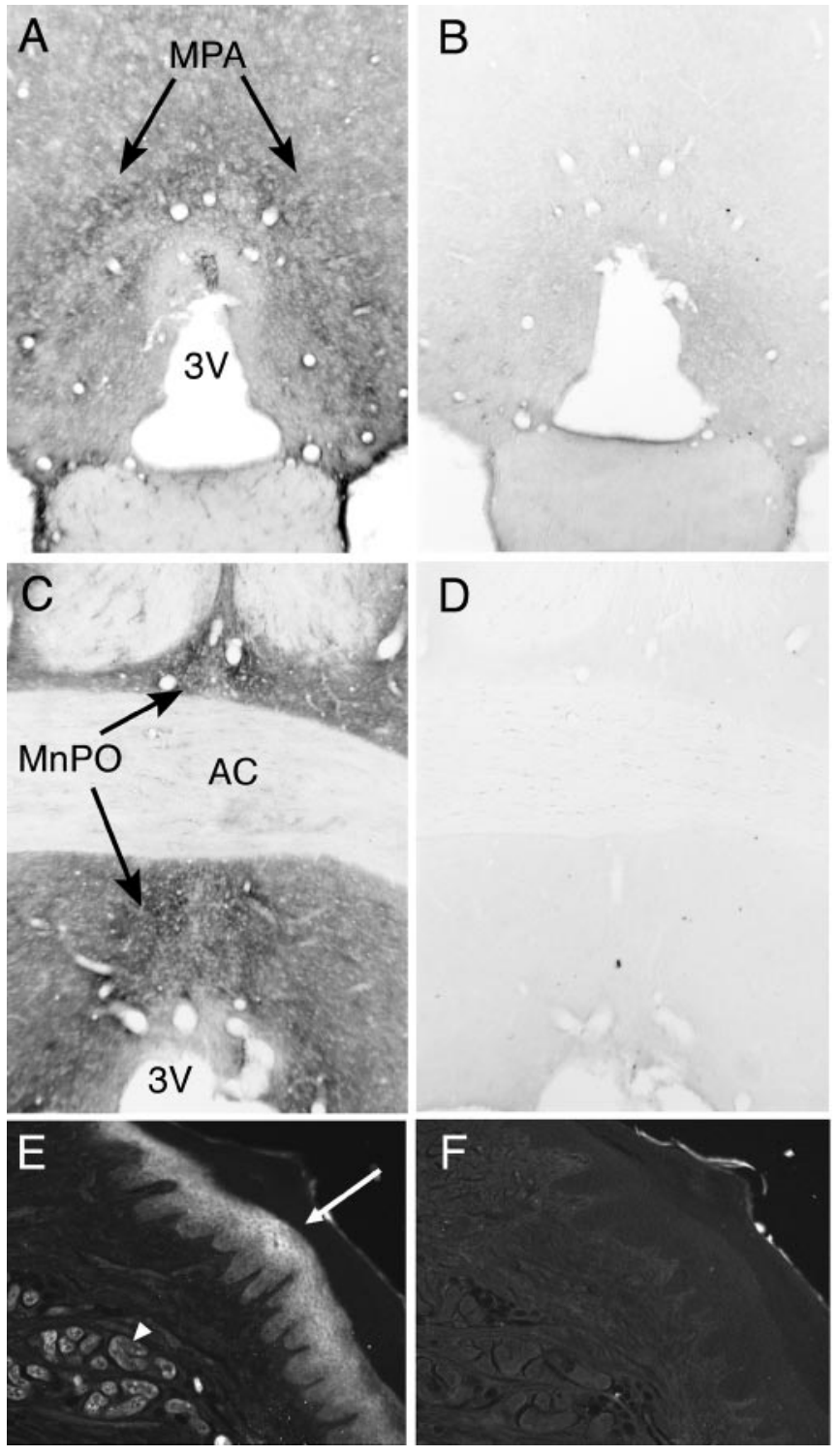

Figure 6. Immunohistological detection of TRPV4 in the anterior hypothalamus and skin keratinocytes. $A-D$, TRPV4-specific diaminobenzidine immunostaining of the MPA $(A, B)$ and $\operatorname{MnPO}(C, D)$ regions of rat hypothalamus. In $B$ and $D$, anti-TRPV4 was predepleted with antigenic TRPV4 peptide. $A C$, Anterior commissure; $3 V$, third ventricle. $E, F$, TRPV4 immunofluorescence in rat plantar skin keratinocytes (arrow) and sweat glands (arrowhead). In $F$, anti-TRPV4 was predepleted with the antigenic TRPV4 peptide.

portion to hypo-osmolarity-evoked responses in those studies in which heat-evoked responses were evaluated in transiently transfected cells (Strotmann et al., 2000; Delany et al., 2001). Other factors that may have impaired the detection of heat-evoked responses include the use of GFP-tagged constructs (Strotmann et al., 2000) or the desensitization of heat-evoked responses during slow temperature ramps with infrequent sampling (Liedtke et al., 2000). Finally, cofactors necessary for heat-evoked activation of TRPV4 might be expressed differentially among host cells.

Single-channel analyses of TRPV1 in excised membrane patches have suggested a direct effect of heat on the open probability of that channel (Tominaga et al., 1998). In the absence of similar analyses of TRPV4, we cannot yet rule out the possibility that this protein is activated indirectly by other heat-stimulated 
proteins, by small molecules liberated in response to heat, or by the physicochemical effects of heat on the plasma membrane. Indeed, an indirect mechanism previously has been suggested for certain heat-evoked responses in cultured sensory neurons (Reichling and Levine, 1997).

Our findings also support the functional integration of thermal and osmotic information at TRPV4. Heat-evoked TRPV4 responses are larger at relatively low osmolarities and virtually ablated at high osmolarities. Previous studies have suggested the converse relationship, namely that the hypo-osmolarity response of TRPV4 is temperature-dependent, with a greater response being observed at 37 than at $22^{\circ} \mathrm{C}$ (Liedtke et al., 2000). These two findings are likely to reflect the same stimulus convergence process. However, our observation that heat-evoked responses of full size can be evoked after desensitization of a hypo-osmolarityevoked response suggests that some degree of independence exists between these two mechanisms of TRPV4 activation. An analogous situation has been observed for TRPV1, in which proton-, heat-, and capsaicin-evoked responses are mechanistically distinguishable, although they interact (Jordt et al., 2000; Jordt and Julius, 2002). As with heat, the mechanism by which hypo-osmolarity activates TRPV4 is unknown. In particular, it is not entirely clear whether TRPV4 senses hypo-osmolarity, per se, the resultant cellular swelling, or a signaling molecule released in response to swelling. Regardless, these findings and the recently demonstrated activation of TRPV4 by phorbol derivatives (Watanabe et al., 2002) suggest that this protein, like TRPV1, can be regulated by an array of partially convergent physical and chemical stimuli.

Under what physiological circumstances might the thermal activation of TRPV4 be relevant? The expression of TRPV4 protein in the MPA and MnPO regions of the anterior hypothalamus, in addition to its temperature-response characteristics, suggests that it might act as a heat transducer in this setting. Electrophysiological studies of organotypic anterior hypothalamic slices have provided evidence for the existence of a heatactivated nonselective cation channel in warm-sensitive neurons, although the molecular identity of that channel is not known (Hori et al., 1999). Such studies also have revealed a complex relationship between thermosensation and osmosensation at the level of individual anterior hypothalamic neurons. Although many warm-sensitive neurons are inhibited by local hyperosmolarity or activated by hypo-osmolarity, others exhibit the opposite relationship (Silva and Boulant, 1984; Travis and Johnson, 1993). Given its dual responsiveness to thermal and osmotic stimuli, TRPV4 might mediate some of these composite effects. Consistent with reported results (Liedtke et al., 2000; Schumacher et al., 2000; Delany et al., 2001), we detected TRPV4 mRNA in peripheral sensory ganglia but failed to observe TRPV4 immunoreactivity in the neuronal cell bodies of these ganglia. Therefore, it is unclear to what extent this protein is expressed in primary afferent neurons or whether it contributes to peripheral heat transduction. We did observe specific TRPV4 immunoreactivity in skin keratinocytes. No direct role in thermosensation has been demonstrated for these cells. Nevertheless, their proximity to the animal's surroundings and to the nerves that transmit thermal and mechanical information to the CNS makes this observation intriguing, especially in light of the report that TRPV1 also is expressed in keratinocytes (Inoue et al., 2002). Given these findings, future studies should be aimed at evaluating the functional significance of TRPV4 heat sensitivity, osmosensitivity, and expression in neuronal and non-neuronal cells by using native preparations. In addition, the polymodal responsiveness of TRPV4 creates an opportunity to use structure-function approaches to explore the biophysical basis of thermosensation and osmosensation. Such studies may reveal why responsiveness to one or both of these modalities is an increasingly common feature of the TRPV subfamily.

\section{REFERENCES}

Boulant J (2000) Role of the preoptic-anterior hypothalamus in thermoregulation and fever. Clin Infect Dis 31:S157-S161.

Caterina MJ, Schumacher MA, Tominaga M, Rosen TA, Levine JD, Julius D (1997) The capsaicin receptor: a heat-activated ion channel in the pain pathway. Nature 389:816-824.

Caterina MJ, Rosen TA, Tominaga M, Brake AJ, Julius D (1999) A capsaicin receptor homologue with a high threshold for noxious heat. Nature 398:436-441.

Delany NS, Hurle M, Facer P, Alnadaf T, Plumpton C, Kinghorn I, See CG, Costigan M, Anand P, Woolf CJ, Crowther D, Sanseau P, Tate SN (2001) Identification and characterization of a novel human vanilloid receptor-like protein, VRL-2. Physiol Genomics 4:165-174.

Hori A, Minato K, Kobayashi S (1999) Warming-activated channels of warm-sensitive neurons in rat hypothalamic slices. Neurosci Lett 275:93-96.

Inoue K, Koizumi S, Fuziwara S, Denda S, Inoue K, Denda M (2002) Functional vanilloid receptors in cultured normal human epidermal keratinocytes. Biochem Biophys Res Commun 291:124-129.

Jordt SE, Julius D (2002) Molecular basis for species-specific sensitivity to "hot" chili peppers. Cell 108:421-430.

Jordt SE, Tominaga M, Julius D (2000) Acid potentiation of the capsaicin receptor determined by a key extracellular site. Proc Natl Acad Sci USA 97:8134-8139.

Liedtke W, Choe Y, Marti-Renom MA, Bell AM, Denis CS, Sali A, Hudspeth AJ, Friedman JM, Heller S (2000) Vanilloid receptorrelated osmotically activated channel (VR-OAC), a candidate vertebrate osmoreceptor. Cell 103:525-535.

McKemy DD, Neuhausser VM, Julius D (2002) Identification of a cold receptor reveals a general role for TRP channels in thermosensation. Nature 416:52-58.

Peier AM, Moqrich A, Hergarden AC, Reeve AJ, Andersson DA, Story GM, Earley TJ, Dragoni I, McIntyre P, Bevan S, Patapoutian A (2002) A TRP channel that senses cold stimuli and menthol. Cell 108:705-715.

Raja SN, Meyer RA, Ringkamp M, Campbell JN (1999) Periphera neural mechanisms of nociception. In: Textbook of pain (Wall PD, Melzack R, eds), pp 11-57. Edinburgh: Churchill-Livingstone.

Reichling DB, Levine JD (1997) Heat transduction in rat sensory neurons by calcium-dependent activation of a cation channel. Proc Natl Acad Sci USA 94:7006-7011.

Schumacher MA, Jong BE, Frey SL, Sudanagunta SP, Capra NF, Levine JD (2000) The stretch-inactivated channel, a vanilloid receptor variant, is expressed in small-diameter sensory neurons in the rat. Neurosci Lett 287:215-218.

Silva NL, Boulant JA (1984) Effects of osmotic pressure, glucose, and temperature on neurons in preoptic tissue slices. Am J Physiol 247:R335-R345.

Strotmann R, Harteneck C, Nunnenmacher K, Schultz G, Plant TD (2000) OTRPC4, a nonselective cation channel that confers sensitivity to extracellular osmolarity. Nat Cell Biol 2:695-702.

Tominaga M, Caterina MJ, Malmberg AB, Rosen TA, Gilbert H, Skinner K, Raumann BE, Basbaum AI, Julius D (1998) The cloned capsaicin receptor integrates multiple pain-producing stimuli. Neuron 21:1-20.

Travis K, Johnson AD (1993) In vitro sensitivity of median preoptic neurons to angiotensin II, osmotic pressure, and temperature. Am J Physiol 264:R1200-R1205.

Watanabe H, Davis JB, Smart D, Jerman JC, Smith GD, Hayes P, Vriens J, Cairns W, Wissenbach U, Prenen J, Flockerzi V, Droogmans G, Benham CD, Nilius B (2002) Activation of TRPV4 channels (hVRL2/mTRP12) by phorbol derivatives. J Biol Chem 277:13569-13577.

Wes P, Chesevich J, Jeromin A, Rosenberg C, Stetten G, Montell C (1995) TRPC1, a human homolog of a Drosophila store-operated channel. Proc Natl Acad Sci USA 92:9652-9656.

Wissenbach U, Bodding M, Freichel M, Flockerzi V (2000) Trp12, a novel Trp-related protein from kidney. FEBS Lett 485:127-134.

Xu XZS, Li HS, Guggino WB, Montell C (1997) Coassembly of TRP and TRPL produces a distinct store-operated conductance. Cell 89:1155-1164.

Zitt C, Obukhov AG, Strubing C, Zobel A, Kalkbrenner F, Luckhoff A, Schultz G (1997) Expression of TRPC3 in Chinese hamster ovary cells results in calcium-activated cation currents not related to store depletion. J Cell Biol 138:1333-1341. 